\title{
Summarising progressive approaches to choosing organisational and technological solutions for the construction of buildings
}

\author{
Sergey Sinenko ${ }^{1}$ and Tatiana Poznakhirko ${ }^{1^{*}}$ \\ ${ }^{1}$ Moscow State University of Civil Engineering, Yaroslavskoeshosse, 26, Moscow, 129337, Russia
}

\begin{abstract}
The article deals with the stages of choosing organisational and technological solutions for the construction of buildings. Three approaches to choosing an optimum organisational and technological solution are presented, compared, and analysed. A chart is given for choosing the organisational and technological solution to erect a building. The most promising approach to finding an optimum is highlighted, namely modelling of the construction of buildings, structures, and complexes as the most effective and justified approach incorporating the best aspects of the first two and offering an opportunity to accomplish the task in the most objective way.
\end{abstract}

\section{Introduction}

The construction of buildings and structures is a complex industrial process characterised not only by complex dynamic tasks, but also by a diversity of decisions that are made. Choosing the best and economically most profitable organisational and technological solutions for erecting buildings and structures is one important task in construction that almost all leading institutes in Russia deal with. The problem becomes important when construction processes are implemented for the first time and when minor quantitative changes are introduced to a design that in most cases lead to change of both the technology and organisation of construction. At the present time, the main stages of choosing an organisational and technological solution for erecting buildings and structures [1-5] are clearly identified, but the conventional approach does not make it possible to solve the problem of a definite choice of the technology and organisation of work because it is not formalised enough; does not offer the opportunity of going over a large number of options; excludes probabilistic assessment of building methods and does not offer the opportunity of taking account of disturbing influences in the necessary degree; makes use of a not-very-objective and crude mechanism for comparing options. Optimization of organizational and technological solutions of SMR production is aimed at choosing the option, which, taking into account local conditions, ensures the maximum reduction of the work execution time with efficient use of material and technical resources. Optimality of solutions is revealed on the basis of variant design and comprehensive analysis of compared options. The essence of optimization

*Corresponding author: top1977@ya.ru 
search is to divide the design process into stages. This allows at each stage to analyse the organizational and technological links between individual elements and to compare the stage variants with each other or with a basic (analog) version. The logical scheme for optimizing organizational and technological solutions is shown in Fig. 3.4. According to the scheme, a series of consecutive calculation and logical operations are performed in each block, the content of which is as follows.

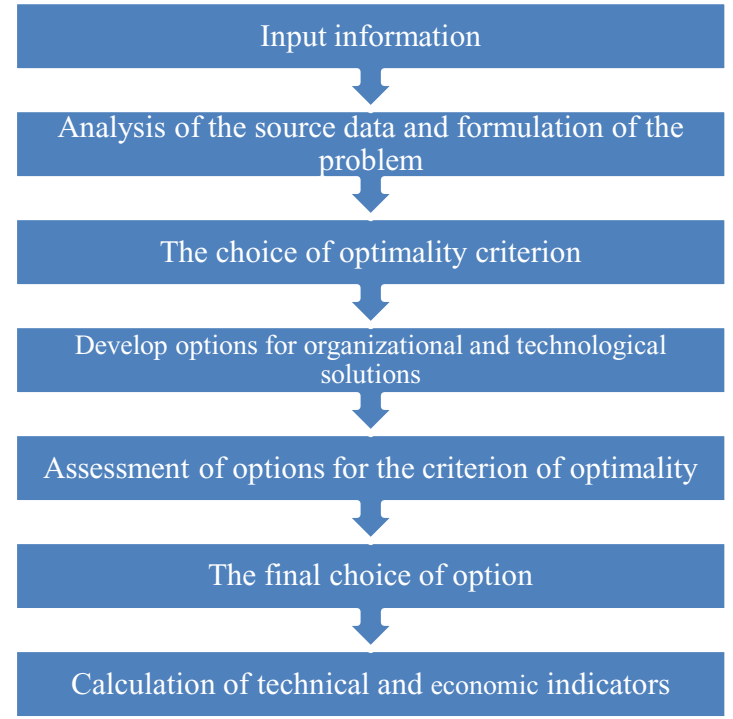

Fig. 1. Scheme of the optimization of organizational and technological solutions.

\section{Methods, results, and discussion}

Today it is possible to isolate several key approaches characterised by specific decisionmaking and leading to the solving of the problem of a rational choice of a construction method.

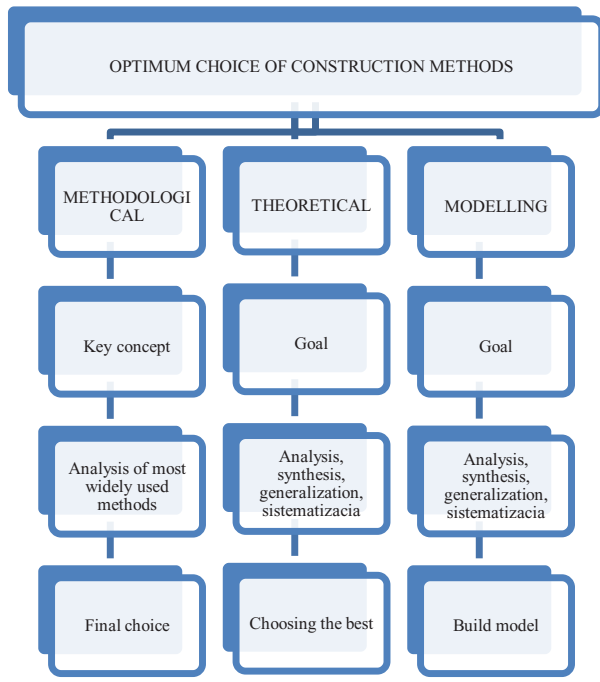

Fig. 2. The scheme of choice of organizational and technological solutions of the construction of buildings and structures. 
The first of these approaches appears in the creative activity of an engineer when he or she tries to choose the best solution under given conditions and finds it by comparing several options, sometimes unconsciously, without a concretely formulated model of the problem of choosing. Research on the choice of rational construction methods, connected with attempts to deal analytically with the problem of finding "the best solution under given conditions," can be regarded as the second approach.

The third approach is connected with the fact that, in view of the introduction of modern computing facilities and technology, in particular BIM, as well as new disciplines in applied mathematics (operational research, systems theory, etc.), the choice of a construction method has become a special case of the application of mathematical programming methods.

BIM (Building Information Modelling) is a technology of information modelling of buildings, a result of the currently adopted conventional computer-aided design (CAD) system. Its main difference from the latter, besides three-dimensional drawing, is the presence of a database in the model that contains detailed information about the technological, technical, architectural, engineering, construction, cost estimate, and economic characteristics of a building. Depending on requirements, the database can be expanded with legal, operational, environmental, and other information, but the most important thing for the case under consideration is a database of flow charts.

ARCHICAD STAR(T) Edition 2017 can be mentioned as one of such instruments. It contains every tool necessary to draw floor plans, sections, elevations, flow charts, and 3D views and to work out BIM projects in detail. STAR(T) Edition 2017 has high performance and considerable improvements for work on project documents. ARCHICAD STAR(T) Edition 2017 is based on the progressive technology of the Information Modelling of Buildings and methods for their construction, which is implemented in ARCHICAD 20. Besides, ARCHICAD STAR(T) Edition makes available most of the functions and solutions present in ARCHICAD 20.

Each of these approaches can be characterised by specific decision-making in the way of solving the problem and by specific methods for choosing the best solution.

Most of widely used developments dealing with the choice of a rational solution for erecting buildings and structures can be regarded as making up the first approach [5].

Also of interest is the analytical approach to solving the problem of choosing a rational organisational and technological solution for erecting a building. There are numerous analytical methods for finding a solution [5] of which a method presented by German engineers is the most illustrative. The method, based on expert opinions, offers an opportunity not only to compare processes allowing for the influence of the combination of construction factors, but also to classify and analyse issues that arise when the best of a large number of available methods has to be chosen. In this case, any construction process is considered from the point of view of achieving one or another goal which in the final analysis can be presented as a system of individual objectives that differ from each other in significance.

As a result of rating the significance of factors influencing construction, a method for erecting the building is chosen that meets certain minimum requirements, allowing for technical and cost conditions. In the present method an attempt is made at quantitative expression of the method properties and at choosing the most appropriate construction method on the basis of a scoring system, with the help of evaluation functions.

The method is considered progressive compared with the first approach to choosing optimum construction technology and organisation, even though insufficiently unbiased.

The third approach to choosing a method for erecting buildings can be characterised by the running of models that result in choosing the best option. When a model is run, there is a need for specific identification of the goal and area of, conditions for, methods and means of the activity as well as the criteria and methods for evaluating the results. 
A mathematical model of erecting buildings or a part of a building offers the opportunity of accurate analysis of the main physical, economic, and organisational characteristics as well as an opportunity to foresee the model behaviour in the case of a deviation from these parameters. Network models are among the first models of the organisation and technology of erecting buildings. Lengthy use of these models has proved their efficiency, but has also identified some drawbacks. The latter result from the imperfection of network models, their insufficient flexibility in a production environment, and their failure to take account of multiple construction technology and organisation options.

The models currently used in this country are too rigid; they exclude construction options with limited resources, and reflect a biased approach on the part of designers.

Research in network planning and management [5] and the use of probabilistic network models in construction indicate that the network model will soon turn into an efficient method enabling evaluation and control of the process of erecting buildings.

Network models are already singled out as a separate subject of special study both in this country and abroad. Particular properties of the mathematical characteristics of network models are one of the main reasons making it advisable to single them out in this manner. The use of these properties offers the opportunity of considerable improvement of the efficiency of the search for rational solutions. Most of the solutions currently beginning to take shape [1-3,6] can be reduced to looking for imitation models that more or less reflect the process of erecting buildings and structures. The latter (models) are based on various subdivisions of mathematics (statistical decision theory, game theory, theory of correlation, etc.).

Examples of such solutions are quite a few. Such are, for instance, the models proposed by R.I. Fokov, N.P. Buslenko, B.F. Beletsky, being more or less correct when applied to choosing organisational and technological solutions for erecting buildings.

The main advantage of using mathematical modelling consists in the fact that not only optimum or suboptimum solutions are identified, but all phenomena are taken into account that arise in certain technical, economic, and organisation conditions.

\section{Conclusion}

Summarising key approaches to choosing a rational organisational and technological solution for erecting buildings and structures enables the following conclusion:

- Each of the above-described three approaches are more or less relevant and justified for certain stages of the choice of optimum organisational and technological solutions for erecting buildings and structures and have practical value;

- Each of these approaches reveals the most acceptable provisions and approach to solving the problem of the preceding developments using the latter at a higher stage of choosing an optimum solution for erecting a building;

- The most promising approach to finding an optimum is modelling of the process of erecting buildings, structures, and complexes as the most effective and justified approach incorporating the best aspects of the first two and offering an opportunity to accomplish the task in the most objective way.

\section{References}

1. S. Sinenko, A. Slavin, Science overview 1, 98-103 (2016)

2. B.V. Zhadanovsky, S.A. Sinenko, M.F. Kuzhin, V.I. Brodsky, B.F. Shirshikov, V.F. Smokin, Y.A. Shesterikov, Developing organisation designs for the construction of industrial buildings and structures: A textbook (ASV, Moscow 2016) 
3. S.A. Sinenko, S.A. Mamochkin, B.V. Zhadanovsky, The basics of the legal framework for construction. Term-paper design (ASV, Moscow, 2016)

4. S.A. Sinenko, S.A. Mamochkin, B.V. Zhadanovsky, T.K. Kuzmina, The basics of the legal framework for construction. A textbook and practical guide (ASV, Moscow, 2016)

5. A.V. Ginzburg, A.A. Volkov, N.A. Garyayev, S.A. Sinenko, Construction design automation systems: a textbook for higher education institutions (MSCU, Moscow, 2014)

6. B.V. Zhadanovsky, M.F. Kuzhin, N.A. Slavin, V.I. Brodsky, B.F. Shirshikov, Organisational and technological solutions for occupational safety and health in construction design: A textbook (ASV, Moscow, 2015)

7. I.L. Abramov, T.Y. Poznakhirko, A. Sergeev, MATEC Web of Conferences 86, 04063 (2016)

8. Construction Regulations 48.13330.2011

9. A.A. Lapidus, I.L. Abramov, Science Bulletin 4, 6-9 (2017)

10. A.A. Lapidus. A.Yu. Berezhny, Bulletin of Moscow State University of Civil Engineering 3, 149-153 (2012)

11. A. A. Lapidus, Bulletin of Moscow State University of Civil Engineering 1, 175-180 (2014)

12. A.A. Lapidus. A.Yu. Berezhny, Building materials, equipment, technologies of the 21st century, 12 (2010)

13. P.P. Oleynik, V.A. Grigoryev, Construction Technology and Organization 2(7), 42-44 (2014)

14. P.P. Oleynik, V.I. Brodsky, Construction Technology and Organization 2(3), 40-43 (2013)

15. P.P. Oleynik, V.I. Brodsky, Construction Technology and Organization 3(4), 35-38 (2013)

16. P.P. Oleynik, Natural and Technical Sciences Journal 10(88), 412-414 (2015)

17. A.A. Lapidus, I.L. Abramov, Science Bulletin 9, 6-9 (2017)

18. A.A. Lapidus. A.N. Makarov, Bulletin of Moscow State University of Civil Engineering 8, 150-160 (2015)

19. S.A. Bolotin, A.Kh. Dadar, I.S. Ptukhina, Engineering and Construction Journal 7, 82$86(2011)$ 